\title{
LINC01224 promotes colorectal cancer progression through targeting miR-485-5p/ MYO6 axis
}

\author{
Jingfeng $\mathrm{Gu}^{1}$, Liang Dong ${ }^{2}$, Yun Wang ${ }^{3}$, Wenjia Nie², Wencong Liư and Ji-an Zhao ${ }^{1^{*}}$ [D
}

\begin{abstract}
Background: Long noncoding RNAs (InCRNAs) are related to colorectal cancer (CRC) development. However, the role and mechanism of IncRNA LINC01224 in CRC development are largely unknown.

Methods: LINC01224, Yin Yang 1 (YY1), microRNA (miR)-485-5p, and myosins of class VI (MYO6) levels were examined using quantitative reverse transcription polymerase chain reaction and western blotting. Functional analyses were processed through CCK-8, colony formation, flow cytometry, transwell, and xenograft analyses. Dualluciferase reporter, chromatin immunoprecipitation (ChIP), RNA immunoprecipitation, and pull-down assays were conducted to analyze the binding interaction.
\end{abstract}

Results: LINC01224 abundance was elevated in CRC tissue samples and cell lines. Elevated LINC01224 might indicate the lower 5-year overall survival in 52 CRC patients. LINC01224 was upregulated via the transcription factor YY1. LINC01224 knockdown restrained CRC cell proliferation, migration, and invasion and increased apoptosis. MiR485-5p was sponged by LINC01224, and miR-485-5p downregulation relieved the influence of LINC01224 interference on CRC progression. MYO6 was targeted via miR-485-5p and regulated via LINC01224/miR-485-5p axis. MiR-485-5p overexpression suppressed CRC cell proliferation, migration, and invasion and facilitated apoptosis. MYO6 upregulation mitigated the role of miR-485-5p. LINC01224 knockdown decreased xenograft tumor growth. Conclusion: YY1-induced LINC01224 regulates CRC development via modulating miR-485-5p/MYO6 axis.

Keywords: Colorectal cancer, YY1, LINC01224, miR-485-5p, MYO6

\section{Introduction}

Colorectal cancer (CRC) is a commonly diagnosed cancer with a high fatality rate around the world [1]. More than 90\% CRCs are adenocarcinoma developing from the glandular epithelial cells of the colon and rectum [2]. Although huge improvements have been made on the pathogenesis and treatment of CRC, the prevention and prognosis of CRC remain unsatisfactory. Hence, exploring novel targets for CRC therapy is necessary.

\footnotetext{
* Correspondence: bairenduizhang2020@163.com

'Department of General Surgery, the First Hospital of Hebei Medical University, No.89 Donggang Road, Shijiazhuang, Hebei, China

Full list of author information is available at the end of the article
}

Long noncoding RNAs (lncRNAs; >200 nucleotides) with diverse cellular functions and lacking proteincoding potential are related to human cancer progression [3]. IncRNAs have become potential targets for the treatment of CRC [4]. Moreover, the roles played via lncRNAs in CRC might be associated with the interaction of microRNAs (miRNAs) and mRNAs [5]. The lncRNA LINC01224 exhibits an oncogenic role in multiple human malignancies [6-9], including CRC [10]. Nevertheless, the repertoire of LINC01224 in CRC progression is largely unclear.

Moreover, LINC01224 promotes carcinogenesis and tumor development through serving as endogenous

(c) The Author(s). 2021 Open Access This article is licensed under a Creative Commons Attribution 4.0 International License, which permits use, sharing, adaptation, distribution and reproduction in any medium or format, as long as you give appropriate credit to the original author(s) and the source, provide a link to the Creative Commons licence, and indicate if changes were made. The images or other third party material in this article are included in the article's Creative Commons licence, unless indicated otherwise in a credit line to the material. If material is not included in the article's Creative Commons licence and your intended use is not permitted by statutory regulation or exceeds the permitted use, you will need to obtain permission directly from the copyright holder. To view a copy of this licence, visit http://creativecommons.org/licenses/by/4.0/ The Creative Commons Public Domain Dedication waiver (http://creativecommons.org/publicdomain/zero/1.0/) applies to the data made available in this article, unless otherwise stated in a credit line to the data. 
sponge for microRNAs (miRNA), including miRNA (miR)-2467 [10]. miRNAs (<25 nucleotides) are classical and versatile noncoding RNAs, and their association with the development, prognosis, and therapy of CRC has been better-demonstrated [11, 12]. MiR-485-5p is suggested as a target for LINC01224 in epithelial ovarian cancer [8], and miR-485-5p could function as an important biomarker for CRC [13]; furthermore, the tumorsuppressive role of miR-485-5p has also been discovered in CRC cells via affecting functional genes [14, 15]. Nevertheless, whether miR-485-5p is relevant to LINC01224 role in CRC remains to be further validated.

Myosins of class VI (MYO6) is a unique actin-based motor protein, which has important roles in regulating multiple cellular processes [16]. Previous reports have suggested an oncogenic role of MYO6 in several human cancers [17-19]. More importantly, MYO6 might contribute to the development of CRC malignancy as well $[20,21]$. However, whether MYO6 can interact with LINC01224 and miR-485-5p is uncertain.

Thereby, we hypothesized that LINC01224 could affect CRC progression through regulating miR-485-5p and MYO6. To confirm this, expression of LINC01224, miR485-5p, and MYO6 was detected in CRC patients and cells, and cellular functions of LINC01224 and miR-485$5 \mathrm{p}$ were measured in CRC cells. Ultimately, the underlying regulatory mechanism was further confirmed via LINC01224/miR-485-5p/MYO6 competing endogenous (ceRNA) pathway and upstream regulation of the transcription factor Yin Yang 1 (YY1), a well-known transcription factor for lncRNAs [22-24]. This research might indicate a novel insight into the pathogenesis of CRC.

\section{Materials and methods Ethics statement}

Each patient signed the written informed consent. This research was approved by the ethics committee of the first hospital of Hebei Medical University, and all procedures were processed following the guidelines of the Helsinki Declaration. Animal experiments were authorized via the Institutional Animal Care and Use Committee of the first hospital of Hebei Medical University and conducted under the National Institutes of Health.

\section{Bioinformatics analysis}

Expression of LINC01224 and MYO6 in colon adenocarcinoma (COAD) and rectum adenocarcinoma (READ) was provided via The Cancer Genome Atlas (TCGA) database (http://www.cancer.gov/tcga) [25]. The binding sites of YY1 and LINC01224 promoter were predicted via JASPAR algorithms (http://jaspar.genereg.net/) [26]. The binding sequences of miR-485-5p in LINC01224 or MYO6 were searched using starBase 2.0 of The Encyclopedia of RNA
Interactomes. platform (http://starbase.sysu.edu.cn/index. php) [27]. The correlation among LINC01224, miR-485-5p, and MYO6 was analyzed on starBase project (http:// starbase.sysu.edu.cn/panGeneCoExp.php). The association between LINC01224 level and overall survival of CRC patients was analyzed on the Gene Expression Profiling Interactive Analysis (GEPIA) server (http://gepia.cancer-pku.cn/ ) $[28]$.

\section{Patient tissue collection}

The CRC and normal control (NC) tissues were harvested from 52 CRC patients in the first hospital of Hebei Medical University from 2011 to 2014. All participants did not receive other treatment before surgery. According to the median of LINC01224 level, these 52 CRC patients were divided into two groups: LINC01224 ${ }^{\text {high }}(n=26)$ and LINC01224 $4^{\text {low }}(n=26)$. The 5 -year overall survival of $52 \mathrm{CRC}$ patients was recorded and analyzed by the Kaplan-Meier survival curve. The correlation between LINC01224 expression and the clinical-pathological features of CRC patients is shown in Table 1.

\section{Cell culture}

CRC cell lines (SW480, HCT116, SW620, and LoVo) and normal colonic mucosa FHC cells were obtained

Table 1 Correlation between LINC01224 expression and the clinical-pathological features of CRC patients

\begin{tabular}{|c|c|c|c|c|}
\hline \multirow[t]{2}{*}{ Characteristic } & \multirow{2}{*}{$\begin{array}{l}\text { All cases } \\
(n=52)\end{array}$} & \multicolumn{2}{|c|}{ LINC01224 expression } & \multirow[t]{2}{*}{$P$-value } \\
\hline & & $\begin{array}{l}\text { High } \\
(n=26)\end{array}$ & $\begin{array}{l}\text { Low } \\
(n=26)\end{array}$ & \\
\hline Age (years) & & & & 0.578 \\
\hline$<60$ & 24 & 11 & 13 & \\
\hline$\geq 60$ & 28 & 15 & 13 & \\
\hline Sex & & & & 0.773 \\
\hline Male & 19 & 9 & 10 & \\
\hline Female & 33 & 17 & 16 & \\
\hline Tumor size, cm & & & & 0.780 \\
\hline$>5$ & 29 & 14 & 15 & \\
\hline$\leq 5$ & 23 & 12 & 11 & \\
\hline Distant metastasis & & & & $0.012^{*}$ \\
\hline Absent & 25 & 8 & 17 & \\
\hline Present & 27 & 18 & 9 & \\
\hline Lymph node metastasis & & & & $0.005^{*}$ \\
\hline No & 22 & 6 & 16 & \\
\hline Yes & 30 & 20 & 10 & \\
\hline TNM stage & & & & 0.575 \\
\hline$|-| \mid$ & 22 & 10 & 12 & \\
\hline III & 30 & 16 & 14 & \\
\hline
\end{tabular}

Chi-square test was used. ${ }^{*} P<0.05$ 
from BeNa Culture Collection (Beijing, China) and maintained in Roswell Park Memorial Institute (RPMI)1640 medium (Thermo Fisher Scientific, Waltham, MA, USA) plus 10\% fetal bovine serum (Gibco, Grand Island, $\mathrm{NY}, \mathrm{USA})$ at $37^{\circ} \mathrm{C}$ and $5 \% \mathrm{CO}_{2}$.

\section{Quantitative polymerase chain reaction (qPCR)}

After lysing in Trizol (Applygen, Beijing, China), the lysates were applied for total RNA extraction as previously reported [29]. A total of $1 \mu \mathrm{g}$ RNA was used for cDNA generation with a reverse transcription kit (Thermo Fisher Scientific). The complex of cDNA, SYBR (Vazyme, Nanjing, China), and primer pairs were used for qPCR with the amplification protocols: $94^{\circ} \mathrm{C}$ for 5 min, 35 cycles of $94^{\circ} \mathrm{C}$ for $15 \mathrm{~s}$, and $60^{\circ} \mathrm{C}$ for $60 \mathrm{~s}$. The primer sequences were provided via Genscript (Nanjing, China) and listed in Table 2. Using U6 or GAPDH as a housekeeping gene, the relative RNA level was analyzed by $2^{-\Delta \Delta \mathrm{Ct}}[30]$.

\section{Cell transfection}

YY1 and MYO6 overexpression vectors were constructed with pcDNA3.1 vector (Thermo Fisher Scientific). The siRNA targeting YY1 (si-YY1), si-NC, shRNA for LINC01224 (sh-LINC01224\#1 and shLINC01224\#2), sh-NC, miR-485-5p mimic, miR-NC mimic, miR-485-5p inhibitor (anti-miR-485-5p), and anti-NC were obtained from Genepharma (Suzhou, China), and their sequences are listed in Table 2. SW620

Table 2 Primers for qPCR and oligonucleotides for transfection

\begin{tabular}{ll}
\hline & Primers (5'-3') \\
\hline LINC01224 & Forward, 5'-ACGTGCACAGACAGCTAAGA-3'; \\
YY1 & Feverse, 5'-GGAGTGACGATCCTGGTGT-3' \\
& Forward, 5'-GGGCCCTTGTCCTGGATAC-3'; \\
MYO6 & Forward, 5'-CCTCTTCACTGGCCCTCATC-3'; \\
& Reverse, 5'-CGGGCTTCCATCCTCCATT-3' \\
miR-485-5p & Forward, 5'-TCGGCAGGAGAGGCTGGCCGT-3'; \\
U6 & Reverse, 5'-AGTGCGTGTCGTGGAGTC-3' \\
& Forward, 5'-TTCGGCAGCACATATACT-3'; \\
GAPDH & Reverse, 5'-CGCTTCACGAATTGCGTGTCA-3' \\
Si-YY1 & Forward, 5'-GCCACTAGGCGCTCAC-3'; \\
si-NC & Reverse, 5'-AGCATCGCCCCACTTGATT-3' \\
sh-LINC01224\#1 & Sense: 5'-UUUACUACUUUAUCAAAACAU-3' \\
sh-LINC01224\#2 & Sense: 5'-AAGACAUUGUGUGUCCGCCTT-3' \\
sh-NC & Sense: 5'-GCACCUAGGUGUUGUCUUA-3'; \\
miR-485-5p mimic & Sense: 5'-GGACGUGCACAGACAGCUA-3 \\
miR-NC mimic & Sense : 5'-AACAGUCGCGUUUGCGACUG-3' \\
Anti-miR-485-5p & 5'-AGAGGCUGGCCGUGAUGCCUUC-3' \\
Anti-NC & 5'-UUCUCCGAACGUGUCACGU-3' \\
\hline
\end{tabular}

and LoVo cells were transfected by using Lipofectamine 3000 (Thermo Fisher Scientific).

\section{Western blotting}

Protein was obtained through RIPA (Beyotime, Shanghai, China). With concentration determination using $\mathrm{BCA}$ kit (Beyotime), $20 \mu \mathrm{g}$ proteins were separated via electrophoresis with sodium dodecyl sulfate, and then electrophoretically transferred onto nitrocellulose membranes (Bio-Rad, Hercules, CA, USA), which were blocked with $3 \%$ bovine serum albumin (Solarbio). Next, special proteins on the membranes were immunoblotted with corresponding primary antibodies for MYO6 (ab230478, 1:500, Abcam, Cambridge, UK), YY1 (ab109228, 1:1,000, Abcam), or GAPDH (ab181602, 1: 3,000 , Abcam), and then with universal secondary antibody (ab205718, 1:10,000, Abcam) for $3 \mathrm{~h}$. Eventually, an immunoblotting signal was developed by exposing to ECL reagent (Beyotime), and the blots were detected via Quantity One v4.6 (Bio-Rad) with GAPDH as control.

\section{Cell Counting Kit (CCK)-8 and colony formation assay}

$1 \times 10^{4}$ cells of differently treated SW620 and LoVo cells were seeded in 96-well plates with four paralleled wells in each group. These cells were further cultured for $72 \mathrm{~h}$ and then added with 10\% CCK- 8 reagent (Beyotime) for $3 \mathrm{~h}$. The optical density was determined at $450 \mathrm{~nm}$ on a microplate reader (Bio-Rad). Cell viability was calculated by the relative OD value with normalization to the control group (100\%). For colony formation assay, 1000 cells/well of SW620 and LoVo cells were seeded in 6well plates for 14-day cultivation. Single-cell-formed colonies were visualized and counted after crystal violet staining (Solarbio, Beijing, China).

\section{Flow cytometry (FCM)}

For cell apoptosis analysis, a group of $1 \times 10^{5}$ cells of SW620 and LoVo cells were harvested for apoptosis and cell cycle analysis respectively using Annexin Vfluorescein isothiocyanate (FITC) apoptosis kit (Abcam) and propidium iodide (PI) FCM kit (Abcam). Briefly, apoptotic cells and cells distributed in cell cycle phases were examined with a flow cytometer (Beckman Coulter, Fullerton, CA, USA).

\section{Transwell assay}

Transwell assay was applied to detect the migratory and invasive abilities. For migration assay, $2 \times 10^{5}$ cells of SW620 and LoVo cells were re-suspended in serum-free RPMI-1640 medium for migration assay with Transwell chamber (BD, Franklin Lakes, NJ, USA), and $5 \times 10^{5}$ cells were for invasion assay with Transwell chamber (BD) coated with Matrigel. In brief, these cells were seeded in the upper chamber, and a complete medium 
was loaded in the lower chamber as an attractant. Transwell systems were incubated in normal cell culture condition for $24 \mathrm{~h}$, and cells transferred onto the lower membranes were visualized and counted after crystal violet staining (Solarbio).

Cells were stained using $0.5 \%$ crystal violet. The images of migratory or invasive cells were captured under a microscope (Nikon, Tokyo, Japan) at magnification $\times 100$.

\section{Dual-luciferase reporter, chromatin immunoprecipitation (ChIP), RNA immunoprecipitation (RIP), and pull-down assays}

LINC01224 promoter containing YY1 binding sites (GCCATC) or mutant type (CGGTAG) were cloned in pGL3-basic vector (Promega, Madison, WI, USA) to generate pGL3-WT1 (pGL3-LINC01224-WT1), pGL3MUT1 (pGL3-LINC01224-MUT1), pGL3-WT2 (pGL3LINC01224-WT2), or pGL3-MUT2 (pGL3-LINC01224MUT2), which were then transfected into SW620 and LoVo cells together with YY1 overexpression vector or vector. The sequences of LINC01224 or MYO6-3'UTR containing miR-485-5p target sites or mutant sites were cloned in pGL3-basic vector to generate LINC01224WT, LINC01224-MUT, MYO6-3'UTR-WT, or MYO63'UTR-MUT, which were transfected in SW620 and LoVo cells together with miR-485-5p mimic or miR-NC. Luciferase activity was detected with a dual-luciferase analysis kit (Promega).

For the ChIP assay, a Magnetic ChIP Kit (Thermo Fisher Scientific) was used. $1 \times 10^{4}$ SW620 and LoVo cells were fixed with $1 \%$ formaldehyde (Solarbio) and then were sonicated for cutting DNA into 500-bp fragments. The DNA samples were precipitated with YY1 antibody (anti-YY1) for $6 \mathrm{~h}$ with anti-IgG as control, in the presence of magnetic beads. The precipitated chromatin DNA was isolated for qPCR.

Cell lysates containing $1 \times 10^{7}$ cells of SW620 and LoVo cells were collected and incubated with Protein A/ G Sepharose ${ }^{\bullet}$ beads provided in the Magna RIP kit (Millipore, Billerica, MA, USA). These beads were preconjugated with the antibody of Ago2 (anti-Ago2) or anti-IgG for $8 \mathrm{~h}$. Similarly, cell lysates containing $1 \times 10^{7}$ cells which were pre-transfected with biotinylated miR485-5p mimic (Bio-miR-485-5p), Bio-NC, biotin-labeled LINC01224 probe, or oligo probe were collected and incubated with the magnetic beads supplied by Magnetic RNA-Protein Pull-Down Kit (Thermo Fisher Scientific). Relative RNA expression levels in precipitated RNA complex on the beads were measured by qPCR.

\section{Xenograft experiment}

sh-LINC01224\#1 or sh-NC-transfected SW620 cells (5 × $10^{6}$ ) were subcutaneously injected in 5-week-old male
BALB/c athymic mice (Vital River Laboratory Animal Technology, Beijing, China) ( $n=6 /$ group$)$. The length and width of each xenograft tumor were monitored every 7 days, and tumor volume was calculated by $0.5 \times$ length $\times$ width $^{2}$ [31]. After 35 days, tumor-bearing mice were euthanized using 5\% isoflurane, and the tumors were detached and weighted. And, tumor tissues were lysed to detect relative levels of LINC01224, miR-4855p, and MYO6.

\section{Statistical analysis}

Data were shown as mean \pm standard deviation from three repeats. The linear correlation between two variables was tested by Pearson's correlation analysis. Association between LINC01224 level and overall survival of patients was evaluated via Kaplan-Meier plot and logrank test. Difference was analyzed by Student's $t$-test or analysis of variance followed by Tukey's post hoc test. Data analysis was performed on GraphPad Prism 6 (GraphPad Inc., La Jolla, CA, USA), and the significance of difference was set as $P<0.05$.

\section{Results}

LINC01224 level is increased and activated by YY1 in CRC To study the importance of LINC01224 in CRC, the expression change of LINC01224 was detected. LINC01224 level was elevated in COAD and READ in the TCGA database (Fig. 1A). Furthermore, LINC01224 abundance was 4-fold higher in CRC than in NC tissues $(n=52)$ (Fig. 1B). Additionally, the patients were classified into LINC01224 $4^{\text {high }}$ and LINC01224 ${ }^{\text {low }}$ groups according to the median value of LINC01224. Clinically, patients with high LINC01224 expression had distant metastasis, lymph node metastasis, and poor overall survival $(P<0.01)$ (Table 1 and Fig. 1C). However, TCGA patients showed that LINC01224 expression might be not associated with overall survival in COAD and READ (Figure S1A). Moreover, LINC01224 abundance was evidently increased in CRC cell lines (SW480, HCT116, SW620, and LoVo) compared with the FHC cell line (Fig. 1D). In order to explore which resulted in the expression change of LINC01224, the upstream transcription factor was analyzed. YY1 was one of the well-known transcription factors for IncRNAs [22-24], and we predicted YY1 could bind to the promoter of LINC01224 using JASPAR algorithms. YY1 mRNA expression was higher in CRC tissues than in the NC group (Fig. 1E). Moreover, YY1 abundance in CRC was positively correlated with LINC01224 (Fig. 1F). In addition, we assessed whether YY1 could change LINC01224 abundance in LoVo and SW620 cells. The efficacy of si-YY1 and YY1 overexpression vector is confirmed in Fig. 1G. 


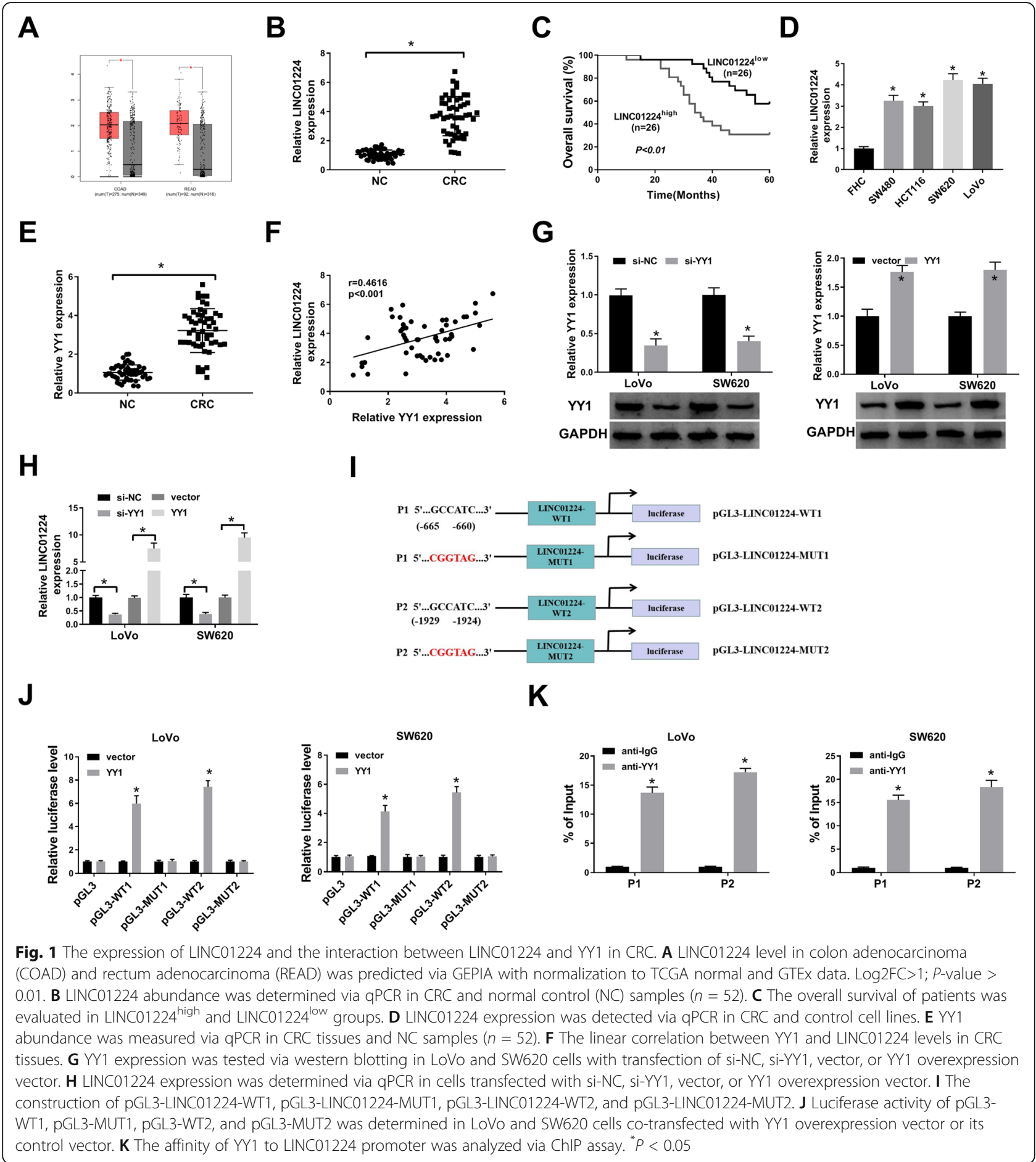

LINC01224 abundance was reduced $60 \%$ by YY1 knockdown and increased more than 7.5 -fold by YY1 overexpression (Fig. 1H). Furthermore, we selected 2 different fragments containing the binding sites between YY1 and LINC01224 with the highest prediction scores (P1 and P2) (Fig. 1I). The dual-luciferase activity analysis showed that YY1 overexpression obviously enhanced the luciferase activity of pGL3WT1 or pGL3-WT2 with the wild-type LINC01224 promoter region, but not the mutant type (Fig. 1J). Besides, the 2 fragments (P1 and P2) were responsible for the affinity of YY1 and LINC01224 (Fig. $1 \mathrm{~K})$. These data suggested that YY1 transcriptionally activated LINC01224 expression. 
LINC01224 silencing represses CRC progression in vitro To study the function of LINC01224 in CRC progression in vitro, LINC01224 abundance in LoVo and SW620 cells was exhausted via shRNAs. LINC01224 level was evidently declined via the transfection of shLINC01224\#1 or sh-LINC01224\#2 compared with shNC, and sh-LINC01224\#1 with higher efficacy was used for further experiments (Fig. 2A). LINC01224 interference evidently reduced cell viability and colony formation ability of LoVo and SW620 cells (Fig. 2B, C). Moreover, LINC01224 silencing evidently induced cell apoptosis and arrested cell cycle (Fig. 2D-F). Additionally, LINC01224 interference clearly restrained the migration and invasion abilities of LoVo and SW620 cells (Fig. 2G, H). These data suggested LINC01224 knockdown constrained CRC progression in vitro.

\section{MiR-485-5p inhibition attenuates the impact of LINC01224 interference in CRC cells}

To probe the network modulated by LINC01224, the target miRNAs were searched via starBase, and a total of 11 potential target miRNAs for LINC01224 were obtained. Among these miRNAs, miR-485-5p was the most enriched by biotin-labeled LINC01224 probe in both LoVo and SW620 cells (Figure S2A and B). Thus, miR485-5p was the most promising candidate as LINC01224 target in CRC. The predicted binding sequences between LINC01224 and miR-485-5p were mutated for luciferase assay (Fig. 3A), and miR-485-5p mimic-mediated overexpression could reduce the luciferase activity of LINC01224-WT but not LINC01224-MUT (Fig. 3B). Furthermore, RNA pull-down analysis displayed LINC01224 could bind with miR-485-5p (Fig. 3C). In addition, both LINC01224 and miR-485-5p were co-enriched by Ago2 RIP (Fig. 3D). Besides, the miR-485-5p level was obviously elevated via LINC01224 knockdown (Fig. 3E). These data indicated miR-485-5p was sponged by LINC01224. To study whether miR-485-5p was involved in LINC01224mediated CRC development, rescue experiments were launched with co-transfection. The addition of anti-miR485-5p lowered miR-485-5p abundance in LoVo and SW620 cells (Fig. 3F), and this downregulation reversed LINC01224 knockdown-mediated effects on cell viability, colony formation ability, apoptosis, and cell cycle (Fig. 3G-K). Furthermore, miR-485-5p inhibition improved migration and invasion abilities in LoVo and SW620 cells with LINC01224 interference (Fig. 3L, M). These findings indicated that LINC01224 modulated CRC progression via miR-485-5p.

\section{MiR-485-5p overexpression inhibits CRC progression via target inhibiting MYO6}

To probe the mechanism mediated via LINC01224/miR485-5p axis, the targets of miR-485-5p were searched using starBase. The target sequences (CAGCCUC) of miR-485-5p and MYO6 are exhibited in Fig. 4A. TCGACOAD (colon adenocarcinoma) and READ (rectum adenocarcinoma) analysis show the overexpression of MYO6 in CRC tumors (http://www.cancer.gov/tcga) (Figure S1B). Moreover, our qPCR data showed the downregulation of miR-485-5p and the upregulation of MYO6 in 52 CRC tumors than paired normal tissues (Figure S1C and 1D). However, there seemed no correlation among LINC01224, miR-485-5p, and MYO6 levels in COAD patients integrated from the TCGA project (Figure S1E). To further validate the potential target relationship between miR-485-5p and MYO6, we performed a luciferase assay. As a result, miR-485-5p mimic addition led to a $60 \%$ decrease of luciferase activity of MYO6-3'UTR-WT, and no reduction on that of MYO63'UTR-MUT (Fig. 4B). Additionally, MYO6 protein expression was sharply decreased in response to miR-4855p mimic transfection (Fig. 4C). Besides, the MYO6 protein level was markedly declined by LINC01224 interference, and it was enhanced by miR-485-5p inhibition (Fig. 4D). In order to study the function of miR-485-5p/ MYO6 axis in CRC development in vitro, LoVo and SW620 cells were transfected with different contents. The MYO6 protein level was increased 1.6-fold by the addition of the MYO6 overexpression vector (Fig. 4E). Moreover, miR-485-5p upregulation evidently reduced cell viability and colony formation ability, and it was reversed by MYO6 elevation (Fig. 4F, G). Furthermore, miR-485-5p upregulation clearly promoted cell apoptosis and cycle arrest, and this effect was weakened by the introduction of MYO6 (Fig. 4H-J). Besides, miR-485-5p overexpression evidently declined cell migration and invasion, and this effect was abated via MYO6 addition (Fig. 4K, L). Collectively, miR-485-5p could repress CRC progression in vitro via regulating MYO6.

\section{LINC01224 knockdown reduces cell growth in vivo}

To further study the role of LINC01224 in CRC progression, LINC01224-silenced SW620 cells were used to establish a xenograft tumor model in mice. After 35 days, the volume and weight of xenograft tumors were obviously lower in the sh-LINC01224\#1 group comparing to the control group (Fig. 5A, B). Furthermore, LINC01224 and MYO6 abundances were markedly higher and miR-485-5p was lower in the tumor tissues with sh-LINC01224\#1 transfection (Fig. 5C, D). These data showed that LINC01224 silencing reduced CRC cell growth in vivo.

\section{Discussion}

CRC is a common malignancy with high incidence ( $10 \%$ of all cancers) and mortality ( $\sim 9 \%$ of all cancers) worldwide [32]. IncRNAs are related to cancer phenotypes in CRC via controlling cellular processes, including 


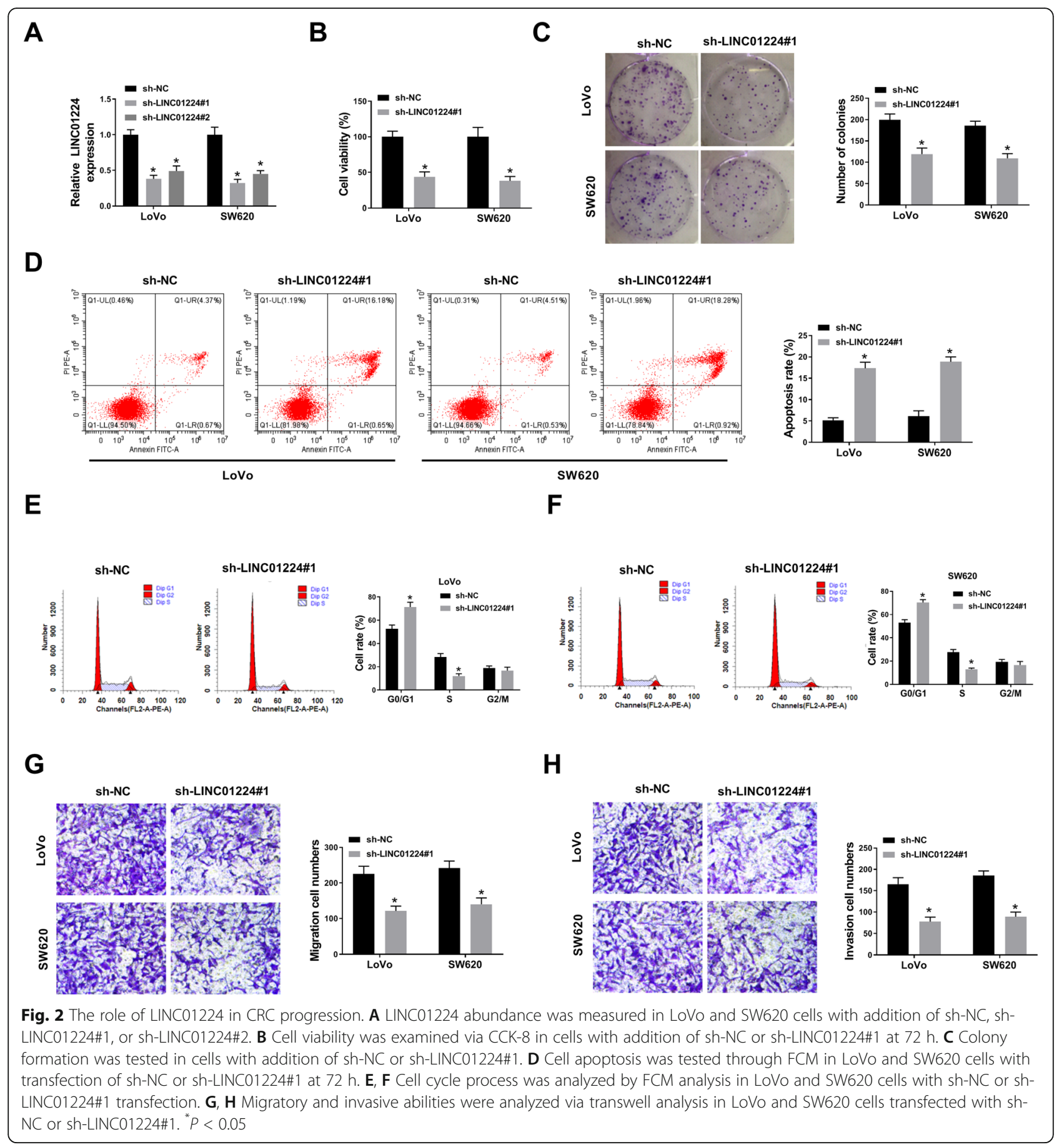

proliferation, apoptosis, migration, and invasion [33]. Mechanically, lncRNAs function as miRNA sponges and mediate competing endogenous (ceRNA) regulatory networks [34-36]. Here, we confirmed the role of LINC01224 in CRC progression and identified the ceRNA pathway of LINC001224/miR-485-5p/MYO6.

Additionally, there is a reported prognostic signature of 6-lncRNA and 12-gene for CRC [37, 38]. Like the data of the TCGA database, we also found LINC01224 abundance was elevated in CRC. Moreover, the increased LINC01224 indicated the poor outcomes of patients. To probe the role of LINC01224 in vitro, we measured the LINC01224 level in CRC cell lines and found that LINC01224 abundance was elevated in CRC cells. Next, we explored which led to the upregulation of LINC001224. YY1 is a zinc finger protein involved in transcriptional control in human cancers [39]. Moreover, YY1 is related to the regulation of IncRNAs [40]. The 
A

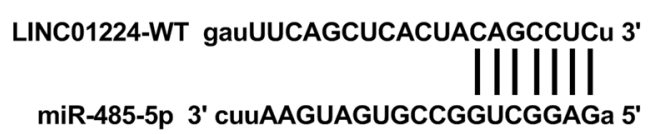

LINC01224-MUT gauUUCAGCUCAC-UAGUCGGAGu 3'

C

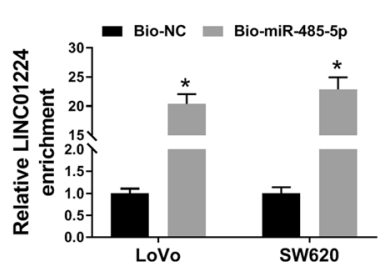

$\mathbf{F}$

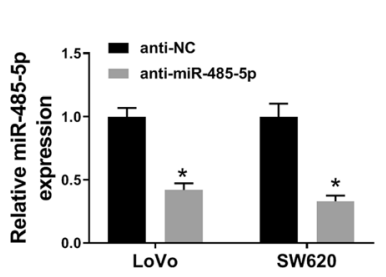

$\mathbf{J}$
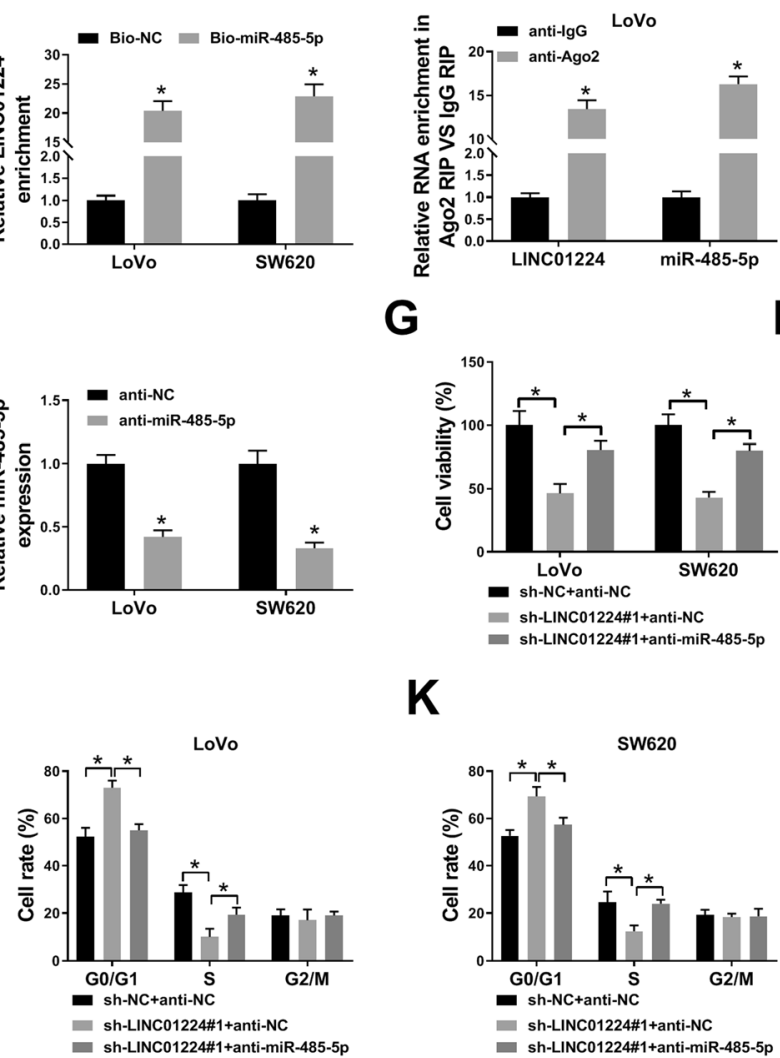

$\mathbf{G}$

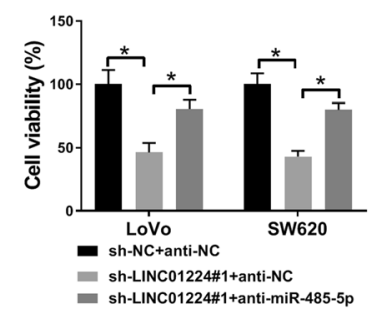

K

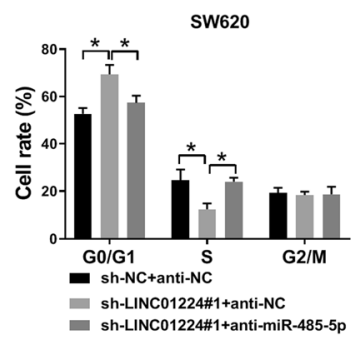

B

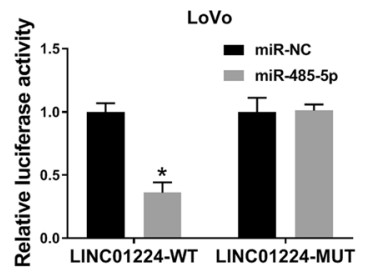

$\mathbf{E}$
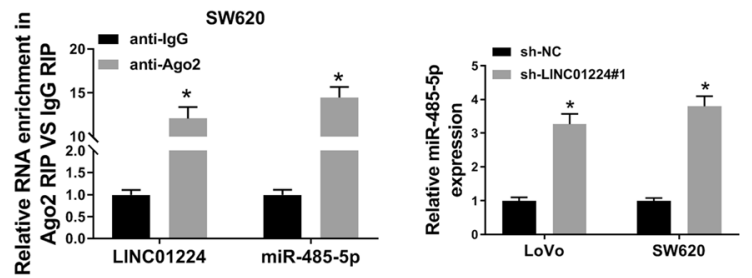

H
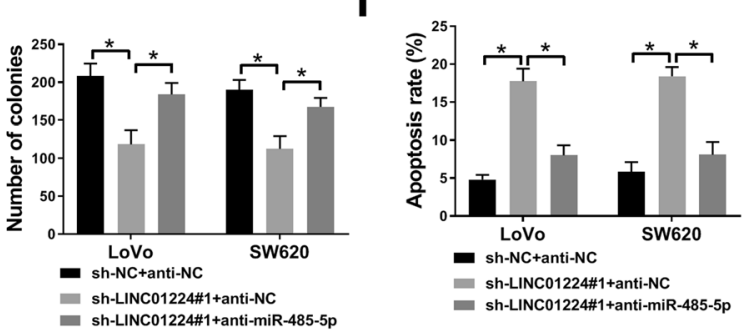

M
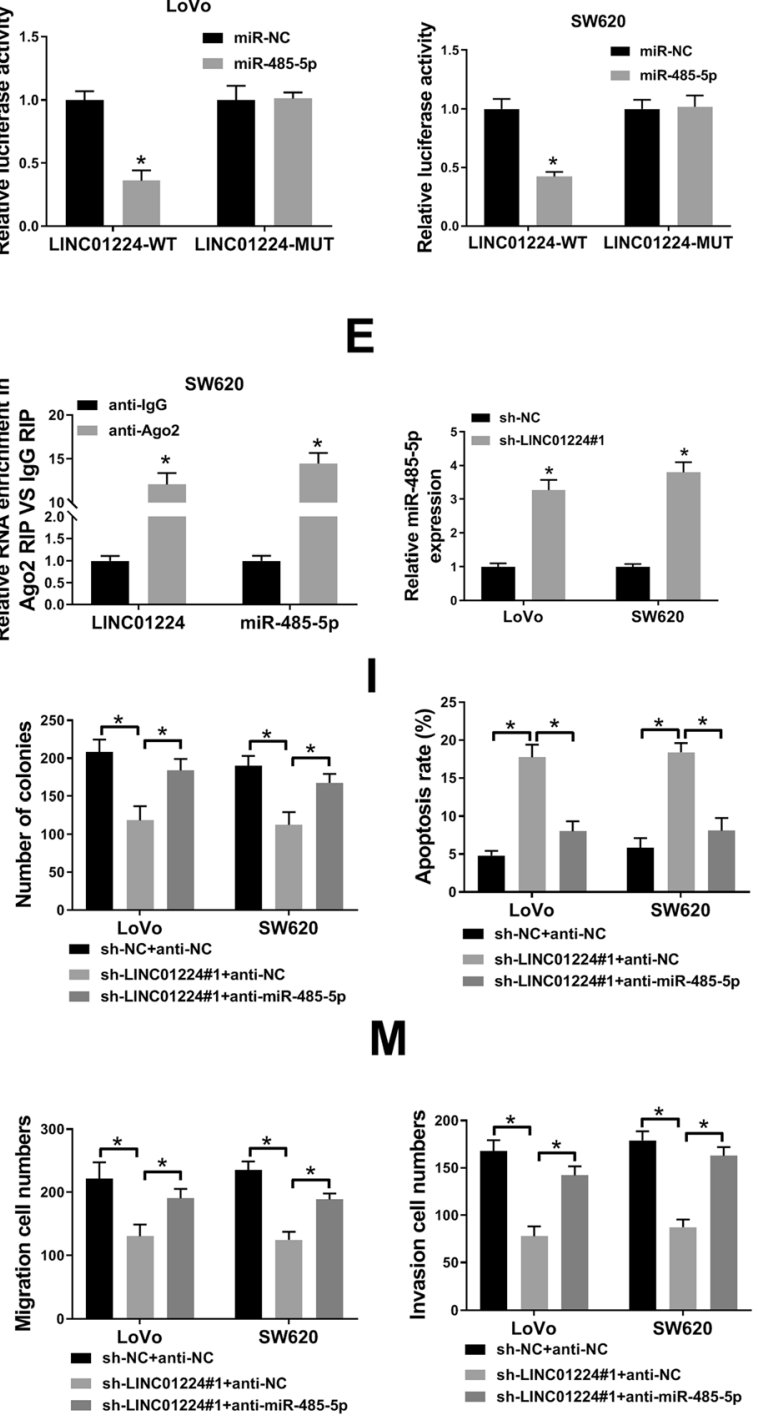

Fig. 3 The relationship between LINC01224 and miR-485-5p in CRC cells. A The target sites of LINC01224 and miR-485-5p. B Luciferase activity of LINC01224-WT and LINC01224-MUT vectors was measured in LoVo and SW620 cells co-transfected with miR-485-5p mimic or miR-NC. C LINC01224 expression was measured after RNA pull-down using Bio-miR-485-5p or Bio-NC. D LINC01224 and miR-485-5p levels were detected after RIP of Ago2 or lgG. E MiR-485-5p level was determined in LoVo and SW620 cells transfected with sh-NC or sh-LINC01224\#1. F MiR-485-5p abundance was determined in cells with transfection of anti-miR-485-5p or anti-NC. Cell viability (G), colony formation (H), apoptosis (I), cycle process $(\mathbf{J}, \mathbf{K})$, migration $(\mathbf{L})$, and invasion $(\mathbf{M})$ were measured by CCK-8 assay, colony formation assay, FCM, and transwell assay in LoVo and SW620 cells co-transfected with sh-NC + anti-NC and sh-LINC01224\#1 + anti-NC or anti-miR-485-5p. ${ }^{*} P<0.05$

increasing evidence has reported that YY1 can activate the expression of lncRNA DDX11 antisense RNA 1 (DDX11-AS1), RAP1 antisense RNA 1 (ARAP1-AS1), and growth arrest-specific transcript 5 (GAS5) in CRC [41-43]. Here, we found that the transcription factor YY1 could bind and induce LINC01224 expression. Moreover, we found LINC01224 silencing constrained CRC cell proliferation and motility, but promoted apoptosis, indicating the anti-CRC role of LINC01224 silencing, which was consistent with the previous study [10].
lncRNA-mediated ceRNA regulatory network is an important mechanism in CRC [44]. Gong et al. have proposed a regulatory network of LINC001224/miR-330$5 \mathrm{p} /$ checkpoint kinase 1 (CHEK1) in hepatocellular carcinoma [7]. Chen et al. claimed that LINC001224 sponged miR-2467 to promote CRC progression [10]. To explore an additional regulatory network, we validated miR-485-5p could be sponged via LINC01224. Multiple reports suggested miR-485-5p suppresses cancer progression of CRC [13, 14, 45, 46]. Similarly, our 


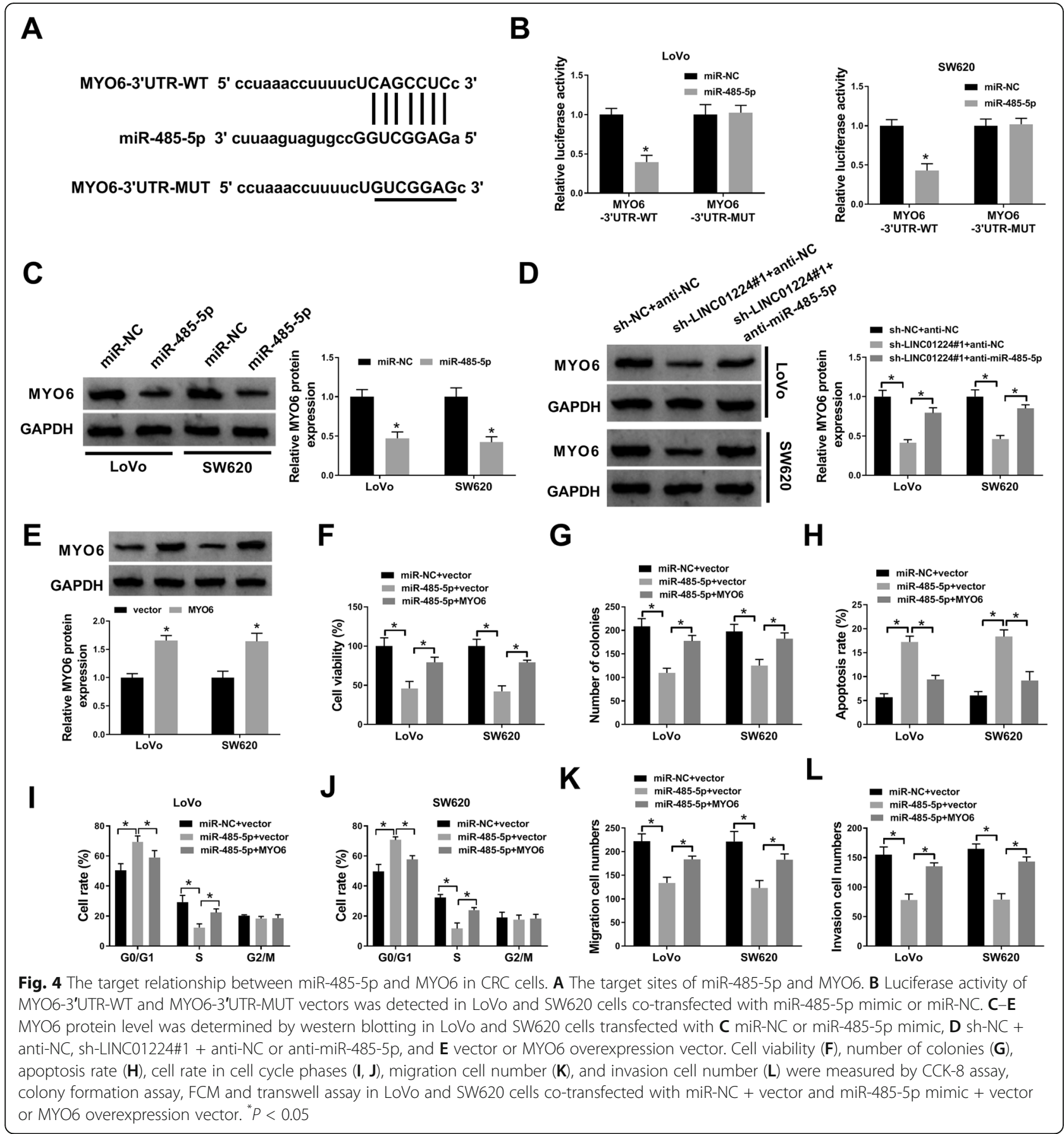

study also found that miR-485-5p addition could repress CRC progression. Additionally, miR-485-5p inhibition abated the cellular functions of LINC01224 depletion on CRC progression, implying that LINC01224 regulated CRC progression via mediating miR-485-5p.

Moreover, we further identified MYO6 was targeted via miR-485-5p. Previous studies have indicated that MYO6 can facilitate CRC malignancy via boosting cell viability, cell cycle, cell motility, and glycolysis [20, 21, 47-50]. These reports suggested that MYO6 played an oncogenic role in CRC. Our research also validated the function of MYO6 via rescuing miR-485-5p-mediated suppressive role in CRC progression. Furthermore, we found that MYO6 expression was regulated via LINC01224 and miR-485-5p, suggesting that LINC01224 could target MYO6 with miR-485-5p as a crosstalk. The preclinical models are helpful to understand the pathogenesis of CRC [51]. Hence, to better expound LINC01224 role in CRC, we established the subcutaneously xenograft model using CRC cells. We found that 


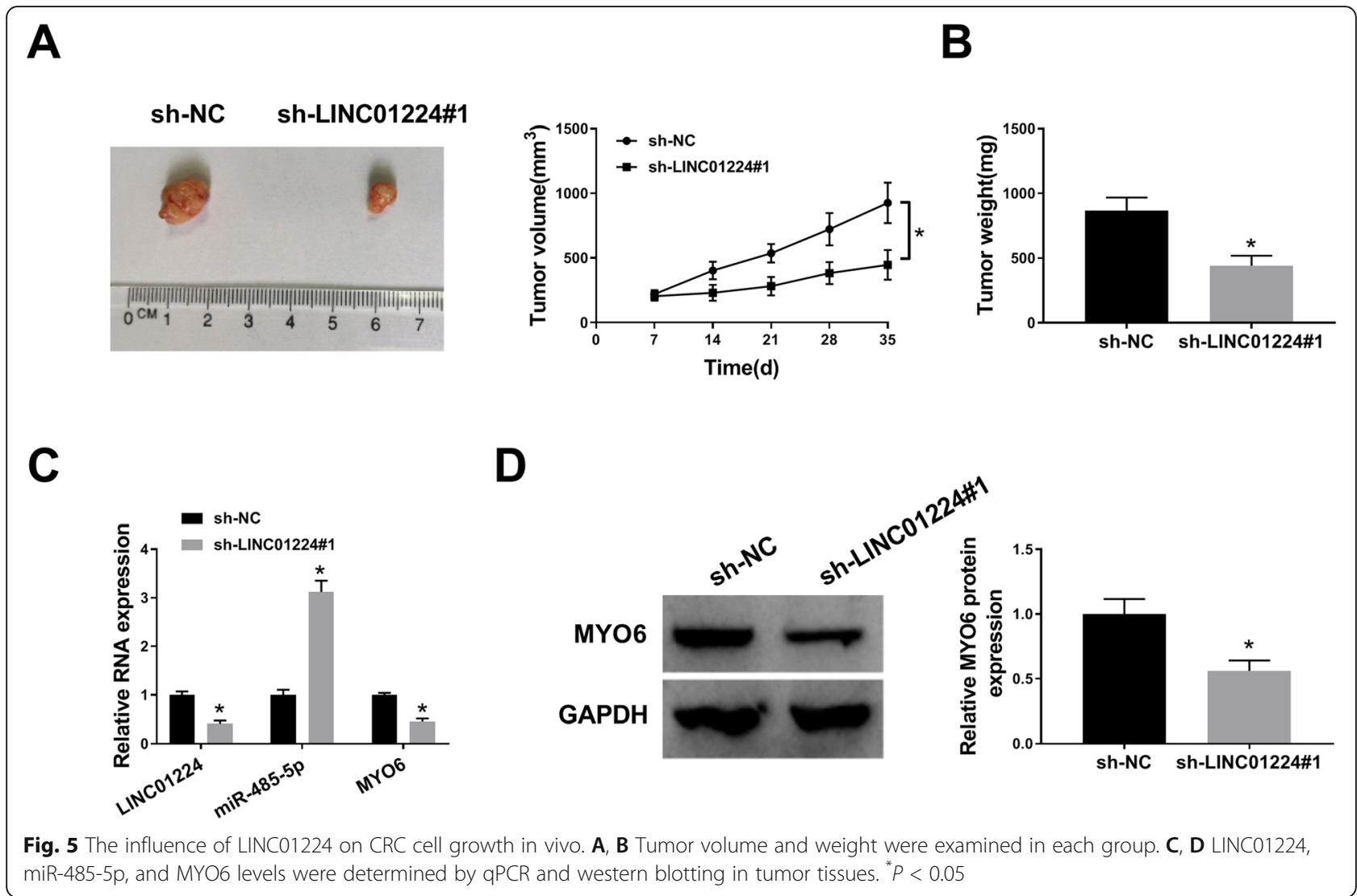

LINC01224 knockdown decreased xenograft tumor growth, indicating the anti-CRC role of LINC01224 knockdown in vivo.

However, there are still some limitations in our study. Firstly, the sample size is 52 for the 5 -year overall survival analysis of CRC patients. Secondly, starBase predicts other miRNAs and genes, which can be targeted by LINC01224 and miR-485-5p, and other pathways in CRC progression can be explored.

In conclusion, LINC01224 knockdown represses CRC progression both in vitro and in vivo partly by regulating miR-485-5p and MYO6 via the ceRNA axis. Our study deepens our understanding on CRC pathogenesis. The study also indicates that LINC01224 high level may act as an unfavorable marker for the overall survival of these 52 CRC patients.

\section{Abbreviations}

IncRNAs: Long noncoding RNAs; CRC: Colorectal cancer; YY1: Yin Yang 1; MYO6: Myosins of class Vl; ChIP: Chromatin immunoprecipitation

\section{Supplementary Information}

The online version contains supplementary material available at https://doi. org/10.1186/s12957-021-02389-x.

Additional file 1: Figure S1. The enrichment of miRNAs in biotin labelled LINC01224 probe in CRC cells. (A, B) RNA pull-down assay and
qPCR determined relative miRNA expression in LINC01224 probe and oligo probe-mediated pull-down contents in LoVo and SW620 cells. ${ }^{* * *} P$ $<0.001$.

Additional file 2: Figure S2. Expression analysis of LINC01224, miR-485$5 p$ and MYO6 in CRC patients. (A) GEPIA database showed the overall survival of COAD and READ patients (integrated from TCGA project) with High and Low LINC01224 level. (B) MYO6 level in COAD and READ was predicted via GEPIA with normalization to TCGA normal and GTEx data. Log2FC $>1$; $p$-value $>0.01$. (C, D) Relative miR-485-5p and MYO6 expression was determined via GPCR in CRC and normal control (NC) samples ( $n=$ 52). (E) StarBase 3.0 project analyzed the correlation among LINC01224, miR-485-5p and MYO6 levels in CRC patients (integrated from TCGA project). ${ }^{*} P<0.05$.

\section{Acknowledgements}

None.

Authors' contributions

LD and YW designed and performed the research; WN, WL, and JZ analyzed the data; JG wrote the manuscript. All authors read and approved the final manuscript.

Funding

Expression of JAK2/STAT3 signal pathway in hepatocarcinoma and the intervention of curcumin analogue

Supported project of key scientific and technological research plan of medical science research in Hebei Province (NO:20190485)

Availability of data and materials Not applicable. 


\section{Declarations}

\section{Ethics approval and consent to participate}

Written informed consents were obtained from all participants, and this study was permitted by the Ethics Committee of the First Hospital of Hebei Medical University.

\section{Consent for publication}

Not applicable.

\section{Competing interests}

The authors declare that they have no competing interests.

\section{Author details}

'Department of General Surgery, the First Hospital of Hebei Medical University, No.89 Donggang Road, Shijiazhuang, Hebei, China. ${ }^{2}$ Department of Medical Service, the First Hospital of Hebei Medical University, Shijiazhuang, China. ${ }^{3}$ Department of Emergency, the First Hospital of Hebei Medical University, Shijiazhuang, China. ${ }^{4}$ Department of Ultrasonography, the First Hospital of Hebei Medical University, Shijiazhuang, China.

Received: 7 April 2021 Accepted: 31 August 2021

\section{Published online: 17 September 2021}

\section{References}

1. Dekker E, Tanis PJ, Vleugels JLA, Kasi PM, Wallace MB, et al. Lancet. 2019; 394(10207):1467-80. https://doi.org/10.1016/S0140-6736(19)32319-0.

2. Keum N, Giovannucci E. Global burden of colorectal cancer: emerging trends, risk factors and prevention strategies. Nat Rev Gastroenterol Hepatol 2019;16(12):713-32. https://doi.org/10.1038/s41575-019-0189-8.

3. Yao RW, Wang Y, Chen LL. Cellular functions of long noncoding RNAs. Nat Cell Biol. 2019;21(5):542-51. https://doi.org/10.1038/s41556-019-0311-8.

4. Jothimani G, Sriramulu S, Chabria Y, Sun XF, Banerjee A, Pathak S. A review on theragnostic applications of microRNAs and long non-coding RNAs in colorectal cancer. Curr Top Med Chem. 2018:18(30):2614-29. https://doi. org/10.2174/1568026619666181221165344

5. Tang XJ, Wang W, Hann SS. Interactions among IncRNAs, miRNAs and mRNA in colorectal cancer. Biochimie. 2019;163:58-72.

6. Li H, Gao C, Liu L, Zhuang J, Yang J, Liu C, et al. 7-InCRNA assessment model for monitoring and prognosis of breast cancer patients: based on Cox regression and co-expression analysis. Front Oncol. 2019;9:1348.

7. Gong D, Feng PC, Ke XF, Kuang HL, Pan LL, Ye Q, et al. Silencing long noncoding RNA LINC01224 inhibits hepatocellular carcinoma progression via microRNA-330-5p-induced inhibition of CHEK1. Mol Ther Nucleic Acids. 2020;19:482-97.

8. Xing S, Zhang Y, Zhang J. LINC01224 exhibits cancer-promoting activity in epithelial ovarian cancer through microRNA-485-5p-mediated PAK4 upregulation. Onco Targets Ther. 2020;13:5643-55.

9. Sun H, Yan J, Tian G, Chen X, Song W. LINC01224 accelerates malignant transformation via MiR-193a-5p/CDK8 axis in gastric cancer. Cancer Med. 2021;10(4):1377-93. https://doi.org/10.1002/cam4.3726

10. Chen L, Chen W, Zhao C, Jiang Q. LINC01224 promotes colorectal cancer progression by sponging miR-2467. Cancer Manag Res. 2021:13:733-42.

11. Sarvizadeh M, Malekshahi ZV, Razi E, Sharifi H, Moussavi N, Taghizadeh M. MicroRNA: a new player in response to therapy for colorectal cancer. J Cell Physiol. 2019;234(6):8533-40. https://doi.org/10.1002/jcp.27806.

12. Huang Y, Zhu N, Chen T, Chen W, Kong J, Zheng W, et al. Triptolide suppressed the microglia activation to improve spinal cord injury through miR-96/IKKbeta/NF-kappaB pathway. Spine (Phila Pa 1976). 2019;44(12): E707-E14. https://doi.org/10.1097/BRS.0000000000002989.

13. Pan Y, Qin J, Sun H, Xu T, Wang S, He B. MiR-485-5p as a potential biomarker and tumor suppressor in human colorectal cancer. Biomark Med. 2020;14(3):239-48. https://doi.org/10.2217/bmm-2019-0534.

14. Li J, Xu J, Yan X, Jin K, Li W, Zhang R. MicroRNA-485 plays tumoursuppressive roles in colorectal cancer by directly targeting GAB2. Oncol Rep. 2018:40(1):554-64. https://doi.org/10.3892/or.2018.6449.

15. O'Mara TA, Glubb DM, Amant F, Annibali D, Ashton K, Attia J, et al. Identification of nine new susceptibility loci for endometrial cancer. Nat Commun. 2018;9(1):3166.
16. de Jonge JJ, Batters C, O'Loughlin T, Arden SD, Buss F. The MYO6 interactome: selective motor-cargo complexes for diverse cellular processes. FEBS Lett. 2019;593(13):1494-507. https://doi.org/10.1002/1873-3468.13486.

17. Wang Z, Ying M, Wu Q, Wang R, Li Y. Overexpression of myosin VI regulates gastric cancer cell progression. Gene. 2016;593(1):100-9. https://doi.org/10.1 016/j.gene.2016.08.015

18. Lei C, Du F, Sun L, Li T, Li T, Min Y, et al. miR-143 and miR-145 inhibit gastric cancer cell migration and metastasis by suppressing MYO6. Cell Death Dis. 2017;8(10):e3101.

19. Yang Q. MicroRNA-5195-3p plays a suppressive role in cell proliferation, migration and invasion by targeting MYO6 in human non-small cell lung cancer. Biosci Biotechnol Biochem. 2019;83(2):212-20. https://doi.org/10.1 080/09168451.2018.1540288.

20. You W, Tan G, Sheng N, Gong J, Yan J, Chen D, et al. Downregulation of myosin $\mathrm{VI}$ reduced cell growth and increased apoptosis in human colorectal cancer. Acta Biochim Biophys Sin (Shanghai). 2016;48(5):430-6. https://doi.org/10.1093/abbs/gmw020.

21. Guo X, Zhang Y, Liu L, Yang W, Zhang Q. HNF1A-AS1 regulates cell migration, invasion and glycolysis via modulating miR-124/MYO6 in colorectal cancer cells. Onco Targets Ther. 2020;13:1507-18.

22. Yan Z, Yang Q, Xue M, Wang S, Hong W, Gao X. YY1-induced IncRNA ZFPM2-AS1 facilitates cell proliferation and invasion in small cell lung cancer via upregulating of TRAF4. Cancer Cell Int. 2020;20:108.

23. You Q, Yao Y, Wu J, Cheng C, Li Y, Yuan H. YY1-induced IncRNA DSCR8 promotes the progression of ovarian cancer via miR-3192-5p/YY1 axis. Biomed Pharmacother. 2020;129:110339.

24. Yang Z, Ding H, Pan Z, Li H, Ding J, Chen Q. YY1-inudced activation of IncRNA DUXAP8 promotes proliferation and suppresses apoptosis of triple negative breast cancer cells through upregulating SAPCD2. Cancer Biol Ther. 2021;22(3):216-24. https://doi.org/10.1080/15384047.2021.1881201.

25. Fan X, Liu L, Shi Y, Guo F, Wang H, Zhao X, et al. Integrated analysis of RNAbinding proteins in human colorectal cancer. World J Surg Oncol. 2020; 18(1):222. https://doi.org/10.1186/s12957-020-01995-5.

26. Fornes $\mathrm{O}$, Castro-Mondragon JA, Khan A, van der Lee R, Zhang X, Richmond PA, et al. JASPAR 2020: update of the open-access database of transcription factor binding profiles. Nucleic Acids Res. 2020;48(D1):D87-92. https://doi. org/10.1093/nar/gkz1001

27. Li JH, Liu S, Zhou H, Qu LH, Yang JH. starBase v2.0: decoding miRNA-ceRNA, miRNA-ncRNA and protein-RNA interaction networks from large-scale CLIPSeq data. Nucleic Acids Res. 2014;42(Database issue):D92-7. https://doi.org/1 $0.1093 /$ nar/gkt1248

28. Tang Z, Li C, Kang B, Gao G, Li C, Zhang Z. GEPIA: a web server for cancer and normal gene expression profiling and interactive analyses. Nucleic Acids Res. 2017;45(W1):W98-W102. https://doi.org/10.1093/nar/gkx247.

29. Chomczynski P, Sacchi N. The single-step method of RNA isolation by acid guanidinium thiocyanate-phenol-chloroform extraction: twenty-something years on. Nat Protoc. 2006;1(2):581-5. https://doi.org/10.1038/nprot.2006.83.

30. Livak KJ, Schmittgen TD. Analysis of relative gene expression data using real-time quantitative $P C R$ and the 2(-delta delta $C(T)$ ) method. Methods. 2001;25(4):402-8. https://doi.org/10.1006/meth.2001.1262

31. Euhus DM, Hudd C, LaRegina MC, Johnson FE. Tumor measurement in the nude mouse. J Surg Oncol. 1986;31(4):229-34. https://doi.org/10.1002/jso.2 930310402.

32. Jung G, Hernandez-Illan E, Moreira L, Balaguer F, Goel A. Epigenetics of colorectal cancer: biomarker and therapeutic potential. Nat Rev Gastroenterol Hepatol. 2020;17(2):111-30. https://doi.org/10.1038/s41575-019-0230-y.

33. Ragusa M, Barbagallo C, Statello L, Condorelli AG, Battaglia R, Tamburello L, et al. Non-coding landscapes of colorectal cancer. World J Gastroenterol. 2015;21(41):11709-39. https://doi.org/10.3748/wjg.v21.i41.11709.

34. Hu X, Zhang L, Tian J, Ma J. Long non-coding RNA PART1 predicts a poor prognosis and promotes the malignant progression of pancreatic cancer by sponging miR-122. World J Surg Oncol. 2021;19(1):122. https://doi.org/10.11 86/s12957-021-02232-3.

35. Zhang W, Wang Z, Cai G, Huang P. Circ DOCK1 regulates USP11 through miR-132-3p to control colorectal cancer progression. World J Surg Oncol. 2021;19(1):67. https://doi.org/10.1186/s12957-021-02173-x.

36. Liu R, Deng P, Zhang Y, Wang Y, Peng C. Circ_0082182 promotes oncogenesis and metastasis of colorectal cancer in vitro and in vivo by sponging miR-411 and miR-1205 to activate the Wnt/beta-catenin pathway. World J Surg Oncol. 2021;19(1):51. https://doi.org/10.1186/s12957-021-021 64-y. 
37. Gao M, Guo Y, Xiao Y, Shang X. Comprehensive analyses of correlation and survival reveal informative IncRNA prognostic signatures in colon cancer. World J Surg Oncol. 2021;19(1):104. https://doi.org/10.1186/s12957-021-021 96-4.

38. Fang Z, Xu S, Xie Y, Yan W. Identification of a prognostic gene signature of colon cancer using integrated bioinformatics analysis. World I Surg Oncol. 2021;19(1):13. https://doi.org/10.1186/s12957-020-02116-y.

39. Khachigian $L M$. The Yin and Yang of $Y Y 1$ in tumor growth and suppression. Int J Cancer. 2018;143(3):460-5. https://doi.org/10.1002/ijc.31255.

40. Lu L, Sun K, Chen X, Zhao Y, Wang L, Zhou L, et al. Genome-wide survey by ChIP-seq reveals YY1 regulation of lincRNAs in skeletal myogenesis. EMBO J. 2013;32(19):2575-88. https://doi.org/10.1038/emboj.2013.182.

41. Tian JB, Cao L, Dong GL. Long noncoding RNA DDX11-AS1 induced by YY1 accelerates colorectal cancer progression through targeting miR-873/CLDN7 axis. Eur Rev Med Pharmacol Sci. 2019;23(13):5714-29. https://doi.org/10.263 55/eurrev_201907_18309.

42. Ye Y, Gu B, Wang Y, Shen S, Huang W. YY1-induced upregulation of long noncoding RNA ARAP1-AS1 promotes cell migration and invasion in colorectal cancer through the Wnt/beta-catenin signaling pathway. Cancer Biother Radiopharm. 2019;34(8):519-28. https://doi.org/10.1089/cbr.2018.2745.

43. Wang Y, Wu S, Yang X, Li X, Chen R. Association between polymorphism in the promoter region of IncRNA GAS5 and the risk of colorectal cancer. Biosci Rep. 2019;39(4):BSR20190091. https://doi.org/10.1042/BSR20190091.

44. Wang L, Cho KB, Li Y, Tao G, Xie Z, Guo B. Long noncoding RNA (IncRNA)mediated competing endogenous RNA networks provide novel potential biomarkers and therapeutic targets for colorectal cancer. Int J Mol Sci. 2019: 20(22):5758. https://doi.org/10.3390/ijms20225758.

45. Dai J, Xu LJ, Han GD, Sun HL, Zhu GT, Jiang HT, et al. MicroRNA-125b promotes the regeneration and repair of spinal cord injury through regulation of JAK/STAT pathway. Eur Rev Med Pharmacol Sci. 2018;22(3): 582-9. https://doi.org/10.26355/eurrev_201802_14271.

46. Liu J, Zhang J, Wang Z, Xi J, Bai L, Zhang Y. Knockdown of circAPLP2 inhibits progression of colorectal cancer by regulating miR-485-5p/FOXK1 axis. Cancer Biother Radiopharm. 2020. https://doi.org/10.1089/cbr.2019.331 0.

47. Luan Y, Li X, Luan Y, Zhao R, Li Y, Liu L, et al. Circulating IncRNA UCA1 promotes malignancy of colorectal cancer via the miR-143/MYO6 axis. Mol Ther Nucleic Acids. 2020;19:790-803.

48. Wei AW, Li LF. Long non-coding RNA SOX21-AS1 sponges miR-145 to promote the tumorigenesis of colorectal cancer by targeting MYO6. Biomed Pharmacother. 2017:96:953-9.

49. Huang $X$, Shen $X$, Peng L, Mai W, Wan Y, Zhang H. CircCSNK1G1 contributes to the development of colorectal cancer by increasing the expression of MYO6 via competitively targeting miR-455-3p. Cancer Manag Res. 2020;12: 9563-75.

50. Liu Y, Li H, Ye X, Ji A, Fu X, Wu H, et al. Hsa_circ_0000231 knockdown inhibits the glycolysis and progression of colorectal cancer cells by regulating miR-502-5p/MYO6 axis. World J Surg Oncol. 2020;18(1):255. https://doi.org/10.1186/s12957-020-02033-0.

51. Sebolt-Leopold JS. Development of preclinical models to understand and treat colorectal cancer. Clin Colon Rectal Surg. 2018;31(3):199-204. https:// doi.org/10.1055/s-0037-1602240.

\section{Publisher's Note}

Springer Nature remains neutral with regard to jurisdictional claims in published maps and institutional affiliations.

Ready to submit your research? Choose BMC and benefit from:

- fast, convenient online submission

- thorough peer review by experienced researchers in your field

- rapid publication on acceptance

- support for research data, including large and complex data types

- gold Open Access which fosters wider collaboration and increased citations

- maximum visibility for your research: over $100 \mathrm{M}$ website views per year

At BMC, research is always in progress.

Learn more biomedcentral.com/submissions 